\title{
Comparison between Chromogenic Colour Agar and Biochemical Tests in the Identification of Causative Organism of Bacteriuria Associated with Urinary Schistosomiasis, in Abu Rukba Village, White Nile State (Sudan)
}

\author{
Husameldin Abdalla Bakhit ${ }^{1}$, Samah Sid Ahmed Mohamed Salih Elsafi ${ }^{2}$, \\ Mohammed Baha Eldin Ahmed Saad ${ }^{3}$, Alaaeldeen Balal Ahmed ${ }^{4}$, \\ Mohammed Eltoum Hamed Azoz ${ }^{5 *}$ and Mohammed A. Suliman ${ }^{6}$
}

\author{
${ }^{1}$ Faculty of Medical Laboratory Sciences, White Nile University, White Nile State, \\ Kosti City, Sudan \\ ${ }^{2}$ Department of Microbiology Laboratory, National Health Insurance, Microbiology \\ Laboratory, White Nile State, Kosti City, Sudan \\ ${ }^{3}$ Omdurman Ahlia Univesity, Faculty of Medical Laboratory Sciences, Omdurman, Sudan \\ ${ }^{4}$ Department of Microbiology, White Nile University, Faculty of Medical Laboratory Sciences, \\ White Nile State, Kosti City, Sudan \\ ${ }^{5}$ Department of Surgery, Faculty of Medicine, University of El Imam El Mahdi, White Nile \\ State, Kosti City, Sudan \\ ${ }^{6}$ Department of Parasitology and Medical Entomology, Faculty of Medical Laboratory \\ Sciences, University of El Imam El Mahdi, White Nile State, Kosti City, Sudan \\ *Corresponding author
}

\section{A B S T R A C T}

\begin{tabular}{|l|}
\hline K e y w o r d s \\
Urinary \\
Schistosomiasis, \\
Bacteriuria, \\
Chromogenic medium, \\
Biochemical tests
\end{tabular}

Chromogenic medium is culture media for simple and fast detection of bacteria using chromogenic substrates, resulting in the different coloration of certain bacteria colonies. Have several advantages over traditional media, such as cysteine-lactose-electrolyte deficient (CLED) medium, allow for easier recognition of mixed growth, save time, reduce workload and provides higher detection rates. To compare between chromogenic colour agar and biochemical tests in identification of organisms causing bacteriuria that associated with urinary Schistosomiasis. The study was conducted in Abu Rukba village in the White Nile State, Sudan. The study was carried out on the entire population of the village (1029 individuals) during the period of November 2011 and November 2013. Urine samples were collected from study population after having their informed consent, school staff and local administrators. A total of 1029 Mid-stream urine specimens were collected aseptically as possible, in a sterile wide mouth container in the morning and processed by the laboratory within 2 hours of collection, or were kept refrigerated at $4^{\circ} \mathrm{C}$ until delivery to the laboratory and were processed no longer than 4 hours after collection. Each specimen was routinely processed using the centrifugation technique to detect Schistosoma and cultivated by using chromogenic agar and Cystine Lactose Electrolyte Deficient agar plate (CLED) then identified by biochemical tests. Antibiotic susceptibility also was done. Bacterial culture for those who were positive for $S$. haematobium infection (594) revealed growth of Staphylococcus aureus in 116 urine samples (19.5\%), Enterococcus faecalis in 57 (9.6\%), Escherichia coli in 28 (4.7\%), Klebsiella pneumoniae in 20 (3.4\%), Proteus species in $2(0.4 \%)$ and mixed infection in $71(11.9 \%)$. Comparing the chromogenic media results with the biochemical reaction results, $95.5 \%$ of all isolates were recovered on both. The remaining $4.5 \%$ of isolates were different by both tests. Chromogenic medium provided a reliable alternative to CLED medium and biochemical tests for the routine culture of urine specimens. 


\section{Introduction}

Urinary tract disease is trait of infection with Schistosoma haematobium which affects in a diffuse manner the entire genitourinary tract ${ }^{(1)}$. Bacterial infections are often recurrent and important complications of the inactive stage of urinary schistosomiasis which may lead to renal failure (2). In schistosomiasis of the urinary bladder, secondary bacterial infections are common and in men can involve the seminal vesicles, spermatic cord, and to a lesser extent the prostate. In women, infection can involve the cervix and fallopian tubes and can cause infertility ${ }^{(3)}$. Opined that it seems possible that agricultural workers and others who are regularly exposed to contaminated water are occasionally simultaneously infected with both the schistosoma parasite and pathogenic bacteria. In the light of the relative high level of schistosomal and bacterial infection, active assessment and reporting of bacteriuria in urinary schistosomiasis and the complementary incorporation of antibacterial therapy to the integrated morbidity control approach to urinary schistosomiasis deserves emphasis $^{(4)}$.

Chromogenic media is an improved diagnostic medium useful for the isolation, counting and direct presumptive rapid identification of urinary tract pathogens: Escherichia coli (pink), Klebsiella species (green blue), Proteus (brown), Enterococcus faecalis (green-turquoise) and Staphylococcus aureus (creamy white) ${ }^{(5)}$. Chromogenic substrates have proved to be a powerful tool in the identification of microorganisms due to the detection of specific enzymes produced by the target microorganism. These enzymes cleave the chromogenic substrate that points up the microorganism by color differentiation of the grown bacterial. Two different chromogenic substrates are present in this medium. First one is cleaved by $B$-glucosidase, allowing the specific detection of enterococci which form blue or turquoise colonies. The other chromogen is cleaved by ß-galactosidase, which gives E.coli a pink color. Cleavage of both enzymes give the colonies a dark bluepurple color giving Klebsiella species. Tryptophan provides a presumptive indication of tryptophane deaminase activity giving Proteus spp, Morganella spp and Providencia spp a light brown color colonies. ${ }^{(5)}$

CHROMagar ${ }^{\mathrm{TM}}$ medium was evaluated for the detection and differentiate of grampositive and gram-negative pathogenic microorganisms in urine and succeeded in detecting all the urine pathogens that were detected by the reference media (CLED) including gram-negative bacilli, Staphylococci, Streptococci, and yeasts colony. Color and morphology on CHROMagar TM accurately differentiate Escherichia coli, Proteus mirabilis, Proteus vulgaris, Pseudomonas aeruginosa and Acinetobacter $\mathrm{spp}^{(6)}$. Chromogenic medium provides a reliable alternative to CLED medium and can be replaced as primary isolation media for urine culture in clinical laboratories ${ }^{(7)}$. There is no significant differences were found between the isolation rates of urinary pathogens on both CLED and chromogenic media ${ }^{(8)}$.

\section{Objectives}

To compare between chromogenic colour agar and biochemical tests in identification of organisms causing bacteriuria that associated with urinary Schistosomiasis.

\section{Materials and Methods}

This study is prospective community based, carried out from November 2011 to November 2013. The study examined mid-stream urine sample of individuals in Abu Rukba village in the White Nile State. The village is situated 94 Kilometers south west of Kosti city which is 
located 300 Kilometers south to capital of Sudan. This study examined 1029 urine sample of individuals with or without signs of urinary disturbance and infection for evidence of urinary Schistosomiasis as well as evaluated associated bacteriological burden and susceptibility pattern of bacteria isolated.

Mid-stream urine specimens were collected aseptically as possible, in a sterile wide mouth container. Each individual was asked to do some exercise before taking the urine specimen.

Since urine itself is a good culture medium, all specimens were processed by the laboratory within 2 hours of collection, or were kept refrigerated at $4^{\circ} \mathrm{C}$ until delivery to the laboratory and were processed no longer than 4 hours after collection. Whenever possible, urine specimens for culture were collected in the morning.

Diagnosis of urinary Schistosomiasis was conducted using the centrifugation concentration technique. $10 \mathrm{ml}$ of the urine sample were centrifuged at 2000 r. p. m for 5 minutes, and the sediment were then examined for each specimen under the low power of the microscope $(10 \mathrm{x})^{(9)}$.

Each urine samples were cultured on Cysteine Lactose Electrolyte Deficient (CLED) agar plates also cultured on chromogenic agar then incubated aerobically at $37^{\circ} \mathrm{C}$ overnight. Identification of growing bacteria was performed according to standard bacteriological methods (Microscopy and biochemical tests). Microscopy (Gram's stain) it is the routine bacterial stain was used to differentiate Gram-positive bacteria (dark purple in colour) and Gram-negative bacteria (red colour). Biochemical tests were used to identify the isolated microorganisms (Catalase test, coagulase test, DNAase test and mannitol salt agar) were used for identification of Gram positive cocci organism, (Oxidase test, Kligler ion agar, Simmon's citrate agar, Christensen urea agar, and Indol test) for Gram negative rod. Data were summarized and grouped into a master sheet, computed and analyzed with statistical package of social sciences (SPSS) software program (Version 16).

\section{Results and Discussion}

The total number of individuals in this study was $1029,513(49.9 \%)$ were male and 516 $(50.1 \%)$ female. The mean of the age was 14.7 years and the standard deviation was 11.8.

Urine for bacterial infection done for 594 individuals those who were positive for $S$. haematobium infection, the result revealed growth of Staphylococcus aureus in 116 urine samples (19.5\%) and Enterococcus faecalis in 57 (9.6\%) were identical results by both methods (chromogenic agar and biochemical test), Escherichia coli in 28 (4.7\%), 27 samples were identical by both methods, the different one was identified by biochemical as Serratia marcescens. Klebsiella pneumoniaein $20(3.4 \%), 18$ samples were typical by both methods, the other different organisms were identified by biochemical as Enterobacter aerogenes and Citrobacter freundii, Proteus species in $2(0.4 \%)$ and mixed infectionin 71 $(11.9 \%)$ and 300 (50.5\%) were not growth.

Comparing the chromogenic media results with the biochemical reaction results, $95.5 \%$ of all isolates were recovered on both. The remaining $4.5 \%$ of isolates were different by both tests (Table 1 and Table 2). The difference in rates of identification of microorganism was found to be statistically insignificant $(\mathrm{P}$. Value $=0.115)$.

The sensitivity of chromogenic method was $98.97 \%$ and the specificity was 99\% comparing with gold standard method (biochemical test). 
Table.1 Shows the organisms causing bacteriuria in patients with S. haematobium infection (594 urine samples)

\begin{tabular}{|c|c|c|}
\hline Micro-organism & Number positive & Percentage \\
\hline Staphylococcus aureus & 116 & $\mathbf{1 9 . 5 \%}$ \\
\hline Enterococcus faecalis & 57 & $\mathbf{9 . 6 \%}$ \\
\hline Escherichia coli & 28 & $\mathbf{4 . 7 \%}$ \\
\hline Klebsiella pneumoniae & 20 & $\mathbf{3 . 4 \%}$ \\
\hline Proteus species & 2 & $\mathbf{0 . 4 \%}$ \\
\hline Mixed infection & 71 & $\mathbf{1 1 . 9 \%}$ \\
\hline No growth & $\mathbf{3 0 0}$ & $\mathbf{5 0 . 5 \%}$ \\
\hline
\end{tabular}

Table.2 Shows comparison between micro-organisms which were identified by chromogenic media and biochemical reaction

\begin{tabular}{|c|c|c|}
\hline Micro-organism & Chromogenic media & Biochemical reaction \\
\hline Staphylococcus aureus & $116(100 \%)$ & $\mathbf{1 1 6}(\mathbf{1 0 0 \%})$ \\
\hline Enterococcus faecalis & $57(100 \%)$ & $\mathbf{5 7}(\mathbf{1 0 0 \%})$ \\
\hline Escherichia coli & $28(100 \%)$ & $\mathbf{2 7}(\mathbf{9 6 . 4 \%})$ \\
\hline Klebsiella pneumoniae & $20(100 \%)$ & $\mathbf{1 8}(\mathbf{9 0 \%})$ \\
\hline Proteus species & $2(100 \%)$ & $\mathbf{2 ( 1 0 0 \% )}$ \\
\hline Mixed infection & $\mathbf{7 1 ( 1 0 0 \% )}$ & $\mathbf{7 1}(\mathbf{1 0 0 \%})$ \\
\hline
\end{tabular}

By comparing between chromogenic media and biochemical reaction tests in the identification of bacterial species, it was found that $95.5 \%$ of all isolates were recovered on both tests. The remaining $4.5 \%$ of isolates were different, the difference was found in three urine samples, where chromogenic media identified $E$. coli in one sample and two Klebsiella pneumoniae in the other two samples, however, the biochemical tests identified Serratia marcescens, Enterobacter aerogenes and Citrobacter freundii in the three samples. This result is in total agreement with Aspevall, et al., ${ }^{(10)}$ who reported that $95 \%$ of organisms in urine sample were identified by both chromogenic and reference media. Baker, et al., ${ }^{(11)}$ reported that Chromogenic medium provided a reliable alternative to CLED medium for the routine culture of urine specimens. Qaiser, et al., ${ }^{(12)}$ reported that there was $100 \%$ agreement in identification of bacterial species by both media from urine sample, and they concluded that chromogenic media can replace CLED as a primary isolation media for urine culture in clinical laboratories in Pakistan. Retelj and Harlander ${ }^{(13)}$ reported that no significant differences were found between the isolation rates of urinary pathogens on both media. They stated that "we believe that the use of chromogenic media in urine investigations, offers multiple advantages without increasing costs in comparison to procedures using CLED. Eyong et al., ${ }^{(14)}$ reported that $95 \%$ of organisms in urine sample were identified by both chromogenic and reference media (CLED), this result is totally agreement with ours.

E Alamin et al., ${ }^{(15)}$ reported that A 76/200 $(38 \%)$ specimens of urine culture showed bacterial growth while 124/200 (62\%) showed no bacterial growth. The isolated bacteria were E.coli (13.5\%), Staphylococcus aureus 
(7.5\%), Klebsiella pneumoniae (5.5\%), Candida albicans (4\%), Enterococcus fecaellis (3.5\%), Proteus vulgaris (2.5\%), Citrobacter (0.5\%), Proteus mirabilis $(0.5 \%)$, and Provedncia $(0.5 \%)$ in khartoum state which is differ from our results in this series.

Chromogenic media has equal or greater sensitivity of detection, superior detection of mixed cultures as a result of better visualization of different species, and enable shorter times to reporting results. Chromogenic media also provides much more useful information regarding appropriate antibiotic therapy after only 24 hours compared to CLED. The chromogenic media provides trust worthy option to CLED medium and biochemical tests for the routine culture of urine specimens in clinical laboratories with sensitivity and specificity of 98.97\% and 99\% respectively, comparing with gold standard method. Additional researches and studies are needed to provide more information about chromogenic media and its uses in clinical laboratories.

\section{References}

1. Pereira, A. J, Ibarluzea, G. J, Alvarez M. J, Marana, F.M, Gallego, S.A, Larringa, S. J and Bernuy, M.C. Mixed Urogential schistosomiasis. Acta Urologica 1997; 2(3): 272-277.

2. Stamm, W.E and Hooton, T.M. Management of urinary tract infections, in adults. N. Egnl. J. Med.1992; 329: 1328-1334.

3. Mostafa, M.H, Shenita, S.A and O'connur, P.J. Relationship between schistosomiasis and bladder cancer. Clinical Microbiology Reviews 1999; 12(1): 978-11.

4. Adeyeba, O. A. and Ojeaga, S. G. T. Urinary Schistosomiasis and concomitant urinary tract pathogens among school children in Metropohitan
Ibadan, Nigeria. Afr. J. Biomed. Res. 2000; 5: 103-107.

5. Massoud Vosough. An Article on Chromogenic Media. Conda News 2010.

6. Samara, Z., Heifetz, M., Talmor, J., Bain, E. and Bahar, J. Evaluation of use of a new chromogenicagar in detection of urinary tract pathogens. Journal of clinic microbiology, 1998; p. 990-994.

7. Lehman, J. S., Farid, Z., Bassily, S. and Kent, D. C. Renal Function in urinary schistosomiasis. Am. J. Trop. Med. Hyg. 1970; 19 (6), 1001-10069.

8. Laughlin, L.W., Farid, Z., Mansour, N, Edman, D. C. and Higashi, G. I. Bacteriuia urinary Schistosomiasis in Egypt. A prevalence survey. 1978; 27 (5): 916-8.

9. Cheesberough M. District Laboratory Practice in Tropical Countries. (Part 1). Egyptian Edition. The Anglo-Egyptian Bookshop. 2004; 238-239.

10. Aspevall, O., Osterman, B., Dittmer, R., Stén, L., Lindbäck, E. and Forsum, U. Performance of four chromogenic urine culture media after one or two days of incubation compared with reference media. J. Clin. Microbiol. 2002; 40(4): 1500-3.

11. Baker, M., Cheetham, P., Shing, J. and Yau, N. Chromogenic urinary tract infection medium: evaluation and introduction for routine urine culture in a large clinical microbiology laboratory. Br. J. Biomed. Sci. 2002; 58(4): 207-11.

12. Qaiser, S., Zeeshan, M., Jabeen, K., Ahsan, T. and Zafar, A. Comparison of chromogenic urinary tract infection medium with cysteine lactose electrolyte deficient media in a resource limited setting. J. Pak. Med. Assoc. 2011; 61(7): 632-5.

13. Retelj, M. J. and Harlander, T. Chromogenic media for urine cultures 
can be cost-effective. ZdravVestn. 2007; 76: 145-9.

14. Eyong, M. E., Ikepeme, E. E. and Ekarem, E. E. Relationship between Schistosoma haematobium infection and urinary tract infection among children in South Eastern, Niger. Postgrad. Med. J. 2008; 15 (2): 89-93.
15. Murtada E Alamin1, Khalid A Abdelhalim, Maha E Alawad. Etiological Bacteria of Urinary Tract Infections among the Pediatrics in Khartoum Province, Sudan. Journal pharmautical research. www.wjpr.net. 2015. vol 4, issue, 1 .

\section{How to cite this article:}

Husameldin Abdalla Bakhit, Samah Sid Ahmed Mohamed Salih Elsafi, Mohammed Baha Eldin Ahmed Saad, Alaaeldeen Balal Ahmed, Mohammed Eltoum Hamed Azoz and Mohammed A. Suliman. 2019. Comparison between Chromogenic Colour Agar and Biochemical Tests in the Identification of Causative Organism of Bacteriuria Associated with Urinary Schistosomiasis, in Abu Rukba Village, White Nile State (Sudan). Int.J.Curr.Microbiol.App.Sci. 8(08): 175-180. doi: https://doi.org/10.20546/ijcmas.2019.808.021 